\title{
Extraction of Phosphorylated Proteins from Formalin-Fixed Cancer Cells and Tissues
}

\author{
Karl-Friedrich Becker ${ }^{*}, 1$, Hildegard Mack ${ }^{1,2}$, Christina Schott ${ }^{1}$, Susanne Hipp ${ }^{1}$, Anne Rappl ${ }^{1}$, \\ Guido Piontek $^{1}$ and Heinz Hofler ${ }^{1,3}$
}

\author{
${ }^{I}$ Technische Universität München, Institut für Pathologie, Trogerstrasse 18, D-81675 München, Germany \\ ${ }^{2}$ Current address: Cancer Biology Program, Division of Hematology/Oncology, Department of Medicine, Beth Israel \\ Deaconess Medical Center/Harvard Medical School, 77 Avenue Louis Pasteur, NRB 1039, Boston, MA 02115, USA
}

${ }^{3}$ Helmholtz Zentrum München - Deutsches Forschungszentrum für Gesundheit und Umwelt (GmbH), Ingolstädter Landstraße 1, D-85764 Neuherberg, Germany

\begin{abstract}
Deregulated signal pathways are promising targets for cancer therapy as they can drive tumour growth, survival, invasion, and metastasis. In most hospitals around the world, histopathological cancer diagnosis is based upon formalin-fixed and paraffin-embedded (FFPE) tissues. In this study, we evaluated whether phosphorylated signaling proteins can be extracted from cells and tissues after formalin fixation. Three epidermal growth factor (EGF) receptor-positive cell lines were stimulated with EGF for various time points and then either fixed with formalin or left unfixed before protein extraction. Using Western blot, we were able to detect all phosphorylated proteins analyzed, including activated (phosphorylated) extracellular signal-regulated kinase (ERK) 1 and 2. There were no differences between formalin-fixed and unfixed cells. Because ERK1/2 kinases are involved in HER2-mediated signal transduction, we applied our methodology to study ERK1/2 and phospho-ERK1/2 in 20 primary breast cancers using protein lysate microarray technology. We found a statistically significant correlation between HER2 status and ERK1/2 expression; however, activation (phosphorylation) of ERK1/2 was independent of HER2 expression. Our study demonstrates the successful extraction of phosphorylated proteins from FFPE tissue samples. Monitoring phosphorylated signaling proteins in FFPE tissues, as applied here, may allow us in the near future to infer the activity levels of proteins in particular pathways as starting point for the design of individual combination therapy regimens without changing the routine clinical workflow for tissue analysis.
\end{abstract}

\section{INTRODUCTION}

Gene mutations in oncogenes and tumor suppressor genes, as well as associated deregulations of central cellular pathways are the main causes for tumor development and progression. Cancer, therefore, is a genomic as well as a proteomic disease [1]. These pathways - in which receptors, adapter molecules, signal transduction proteins, and other kinds of molecules are involved - regulate a variety of circuits, responsible for diverse cellular functions, such as growth, proliferation, differentiation and survival [2]. Signal transduction occurs via hierarchical phosphorylation and dephosphorylation of key components of the pathways, mirroring the activity of specific nodes within the phosphoproteome. One of the best known mitogen activated protein kinases (MAPK), which has extensively been studied before, is ERK1/2 (extracellular signal-regulated kinase 1 and 2). The functions, regulation, role in cancer, and therapeutic targeting of ERK1/2 have recently been summarized [3]. The MAPK pathway is downstream of the ras proto-oncogene whose activation is associated with development and progression of tumours. As signaling molecules such as MAPK are excellent drug targets and may be used as surrogate

*Address correspondence to this author at the Institut für Pathologie, Technische Universität München, Trogerstrasse 18, D-81675 München, Germany; Tel: ++49-89-4140-4591; Fax: ++49-89-4140-4915;

E-mail: kf.becker@lrz.tum.de markers for predicting response to novel therapeutic approaches, one of the major scientific goals is to characterize in detail the information flow within cells, tissues, organs, and organisms [4-6].

During the past decades, many attempts have been made to unravel possible connections between activated receptor tyrosine kinases and other intracellular oncogenes and pathways in order to identify potential therapeutic target structures [7]. Herceptin and Gleevec, targeting HER2 (ERBB2) and BCR/ABL (and c-KIT, PDGFR) respectively, are examples for the success of these tasks. It is estimated that tumor therapy can be markedly improved in the near future by inhibiting specific deregulated signaling proteins. Unfortunately, there is often a lack of correlation between laboratory studies using cell lines or animal models and clinical studies [8]. The percentage of patients responding to the new inhibitors is often less than 30\% [9]. One of the reasons for this rather low percentage of responders may be that the diagnostic markers currently in use for predicting response are not optimal. In addition, the low predictive value of published preclinical data may be due to the limited number of cell line-based models; these cannot reflect the molecular heterogeneity of tumors defined by histology or stage of disease found among patients.

The portfolio for current routine diagnostic methods is mainly based on immunohistochemistry and fluorescence in situ hybridization (FISH). However, in order to identify 
those patients who will benefit from novel therapeutics, quantitative and multiplexed technologies that can be adapted to routine diagnostic pathology are needed in addition to immunohistochemistry and FISH. Moreover, combinations of molecularly targeted anticancer agents are proposed, some of which are already tested in clinical trials. Obviously, more reliable assays for clinical use for either patient selection and/or measuring pharmacodynamic effects of drugs are required [8].

For the development and application of such methods, Institutes of Pathology will play a major role. We have accepted this challenge and started to establish novel technologies for the precise measurement of disease markers in routine clinical tissues. Because formalin fixation and paraffin embedding (FFPE) is the standard procedure for tissue handling in hospitals world-wide, our focus is on FFPE samples. Very recently, we succeeded in extracting full-length immunoreactive proteins from FFPE tissues, and subsequently used reverse phase protein microarrays to quantify important clinical proteins, including HER2 $[10,11]$. Our vision is that protein networks - rather than single proteins - will be the focus of clinical studies using FFPE tissues and aiming at dissecting the molecular basis of targeted therapies. In this study, we evaluated the extraction efficiency of phosphorylated proteins from formalin-fixed cells in vitro, and for the first time applied the method to analyze ERK1/2, key factors for HER2-mediated signal transduction, in a series of formalin-fixed primary breast cancer tissue samples.

\section{MATERIALS AND METHODOLOGY}

\section{Cell Lines}

The squamous cell carcinoma cell line A431 was used as reported earlier [12]. The human glioblastoma cell lines LN18 and LN-229 were used as described [13]. All cells were cultured in Dulbecco's Modified Eagle's Medium (Invitrogen, Paisley, UK) supplemented with $10 \%$ foetal calf serum (PAA, Coelbe, Germany). Cells were incubated with or without epidermal growth factor for the times indicated and harvested at $80 \%$ confluency. After two washes with ice cold phosphate buffered saline (PBS), cells were divided equally into two sets, one of which was fixed with neutral buffered formalin for $30 \mathrm{~min}$ while the other was incubated on ice for the same time. Following fixation, cells were washed again twice with PBS. The Qproteome FFPE tissue kit buffer (Qiagen GmbH, Hilden, Germany) was used for protein extraction. Fixed and unfixed cells resuspended in Qiagen buffer were incubated for $20 \mathrm{~min}$ at $100^{\circ} \mathrm{C}$ and subsequently for two hours at $80^{\circ} \mathrm{C}$. After centrifugation at $16600 \mathrm{~g}$, the pellets were discarded and the supernatants containing proteins were stored at $-20^{\circ} \mathrm{C}$.

\section{Tissues}

We used 20 human breast carcinomas that had been routinely processed (formalin fixation in $10 \%$ neutral buffered formalin) in the Klinikum rechts der Isar of the Technical University of Munich, Germany, from the years 2005 to 2006. Informed consent of each patient was obtained. The study was approved by the Ethical Committee of the Technical University of Munich, reference number 1288/05. Reference hematoxylin/eosin stained sections of the tissues were histologically verified by an experienced pathologist and areas of interest were indicated with a pen on the slides. Subsequent unstained sections of the same paraffin blocks were used for protein extraction [14]. Protein extraction was performed not later than 2 weeks after cutting the paraffin blocks. Proteins were extracted using a commercial protein extraction system (Qproteome FFPE Tissue Kit, Qiagen, Hilden, Germany) which provides optimized conditions for extracting non-degraded total protein from FFPE tissue sections as described previously [11]. Depending on the sizes of the tumor areas, up to $5 \times 10 \mu \mathrm{m}$ sections were cut from each tissue block. After routine deparaffination of the tissue sections, the areas of interest (as indicated in the hematoxylin/eosin stained reference sections) were scratched from the unstained slides with a needle. The microdissected tissue attached to the needle was transferred into Qproteome FFPE Tissue Kit buffer and incubated for $20 \mathrm{~min}$ at $100^{\circ} \mathrm{C}$ and subsequently kept at $80^{\circ} \mathrm{C}$ for two hours. After centrifugation at $16600 \mathrm{~g}$, the pellets were discarded and the supernatant containing proteins were stored at $-20^{\circ} \mathrm{C}$. Protein concentrations were determined using the microBCA protein assay, according to the instructions provided by the supplier (Cat. No. 23235, Pierce, Rockford, IL, USA).

\section{Antibodies}

Table $\mathbf{1}$ lists the proteins analyzed and antibodies used in Western blotting and/or reverse phase protein microarrays.

Table 1. Proteins Analysed and Antibodies Used in Western Blotting and/or Reverse Phase Protein Microarrays

\begin{tabular}{|c|c|}
\hline Primary Antibodies & Suppliers and Order Details \\
\hline Akt & $\begin{array}{l}\text { \#9272, Cell Signaling Technology, Inc., Danvers, } \\
\text { MA/USA }\end{array}$ \\
\hline alpha-Tubulin & $\begin{array}{l}\text { \#T6199, Sigma-Aldrich Chemie GmbH, } \\
\text { Steinheim, Germany }\end{array}$ \\
\hline beta-Actin & $\begin{array}{l}\text { \#A5441, Sigma-Aldrich Chemie GmbH, } \\
\text { Steinheim, Germany }\end{array}$ \\
\hline EGF-Receptor & $\begin{array}{l}\text { \#2232, Cell Signaling Technology Inc., Danvers, } \\
\text { MA/USA }\end{array}$ \\
\hline $\begin{array}{l}\text { ERK1/2 MAP } \\
\text { Kinase }\end{array}$ & $\begin{array}{l}\text { \#9102, Cell Signaling Technology Inc., Danvers, } \\
\text { MA/USA }\end{array}$ \\
\hline HER2 (ERBB2) & \#A0485, DakoCytomation, Hamburg, Germany \\
\hline $\begin{array}{l}\text { Phosho-ERK1/2 } \\
\text { (Thr202/Tyr204) }\end{array}$ & $\begin{array}{l}\text { \#9101, Cell Signaling Technology Inc., Danvers, } \\
\text { MA/USA }\end{array}$ \\
\hline $\begin{array}{l}\text { Phospho-Akt } \\
\text { (Ser473) }\end{array}$ & $\begin{array}{l}\text { \#9271, Cell Signaling Technology Inc., Danvers, } \\
\text { MA/USA }\end{array}$ \\
\hline $\begin{array}{l}\text { Phospho-EGF- } \\
\text { Receptor (Tyr1086) }\end{array}$ & $\begin{array}{l}\text { \#36-9700, Zymed Laboratories/ Invitrogen Im- } \\
\text { munodetection, South San Francisco, CA/USA }\end{array}$ \\
\hline \multicolumn{2}{|l|}{ Secondary Antibodies } \\
\hline $\begin{array}{l}\text { Mouse IgG-HRP- } \\
\text { Conjugate }\end{array}$ & \#NA931, GE Healthcare, Munic, Germany \\
\hline $\begin{array}{l}\text { Rabbit IgG-HRP- } \\
\text { Conjugate }\end{array}$ & $\begin{array}{l}\text { \#7074, Cell Signaling Technology Inc., Danvers, } \\
\text { MA/USA }\end{array}$ \\
\hline
\end{tabular}

\section{Western Blot}

For Western blot analysis, extracts from the cell lines or tissue samples mentioned above were prepared using the 
Qproteome FFPE Tissue Kit. Total protein was assessed as described above. Twenty micrograms of total protein were used for SDS-polyacrylamid gel electrophoresis (SDSPAGE). Western blot and protein detection were performed as described previously [11].

\section{Reverse Phase Protein Microarrays}

Protein lysate microarrays (also called reverse phase protein microarrays) were generated using a hand-held spotter ("MicroCaster", Whatman/Schleicher and Schuell, Dassel, Germany) according to the instructions of the manufacturers and as described previously [11]. From every protein lysate, 2 dilutions (1:2, 1:4) in Qproteome FFPE Tissue Kit buffer (Qiagen) and a negative control (only Qproteome FFPE Tissue Kit buffer) were prepared and transferred - together with the undiluted samples - into a 96 well plate. For every lysate and every dilution, 4 protein spots were applied onto a nitrocellulose coated glass slide (Cat. No. 10484182, FastSlide, Whatman/Schleicher and Schuell, Dassel, Germany). After a peroxidase blocking step, the primary antibodies were applied at $4{ }^{\circ} \mathrm{C}$ for app. 16 hours. Binding of the primary antibodies was detected using the ECLPlus Western Blot Detection System (Amersham/GE Healthcare Europe $\mathrm{GmbH}$, München, Germany). For estimation of total protein amounts, parallel arrays were stained with Sypro Ruby Protein Blot Stain (Molecular Probes, Eugene, OR, USA) according to the manufacturer's instructions and visualized on an Eagle Eye (Stratagene, La Jolla, CA, USA). Developed films were scanned individually on a scanner (Scanjet 3770 , Hewlett-Packard, Hamburg, Germany) with 1200 dpi and were saved as tif files. Density of protein spots was measured using Scion Image (Scion Corporation, Frederick, Maryland). Relative expression levels of proteins were calculated by normalizing to total protein.

\section{Data Analysis}

The results were analyzed by the Spearman rank correlation test, using SPSS version 15.0 (SPSS, Inc, Chicago, IL). In consideration to the Bonferroni adjustment, $p$ values less than 0.05 were considered statistically significant.

\section{RESULTS}

One of the major goals for clinical researchers is to evaluate the information flow within cells and tissues in order to understand and predict the likelihood of response to novel agents targeting deregulated signaling pathways. Up to now, multiplex detection and quantification of signaling proteins have been performed exclusively using frozen tissues. However, as the routine processing of tissues in hospitals is formalin fixation, we asked whether activated (phosphorylated) signaling proteins can be extracted from formalinfixed cells and clinical tissues and detected by Western blot and protein lysate microarrays.

\section{Detection of Phosphorylated Proteins in Formalin-Fixed and Unfixed Cells in Culture}

It is not known whether posttranslational modifications (in this case: phosphorylations of tyrosine, serine or threonine residues) are retained after formalin fixation and protein extraction. The formal possibility exists that the phosphate moiety may be lost when proteins are extracted from formalin-fixed and paraffin embedded tissues because the method used involves heating the samples to $100^{\circ} \mathrm{C}$ in a special buffer system. For that reason, before clinical tissue samples were analyzed, we characterized phosphorylated proteins in lysates from three human cell lines: A431, LN18, and LN-229. Cells were kept in serum-free medium for 16 hours and then treated with $100 \mathrm{ng} / \mathrm{ml}$ EGF for 0,5 , and 60 minutes. Proteins were extracted from formalin-fixed and unfixed cells in the same manner and subjected to SDSPAGE. The proteins analyzed by Western blot included: EGFR, phospho-EGFR (Tyr1086), ERK1/2, phosphoERK1/2 (Thr202/Tyr204), Akt, and phospho-Akt (Ser473). At the time points 5 and $60 \mathrm{~min}$ after stimulation, all phosphorylated proteins of interest could be clearly detected in at least one of the three cell lines analyzed. Generally, we found a very similar pattern of the proteins in formalin-fixed and unfixed cells (Fig. 1). The clear detection of phosphoAkt in the cell line LN-229 and the low levels of phosphoAkt in the cell lines A431 and LN-18 as determined by Western blot were confirmed by protein lysate microarray analysis (not shown). Although weak signals for phosphoAkt were seen in A431 and LN-18 cells after EGF stimulation using protein arrays, these signals were at the detection limit of the Western blot conditions used here.

\section{Detection of Phosphorylated Proteins in a Series of Breast Cancers}

After having demonstrated successful extraction of phosphorylated proteins from formalin-fixed cells, we then started to analyse tissue samples which are representative for human disease. We analyzed 20 breast cancers that were selected from our archive according to the known HER2 status determined by routine immunohistochemistry (HercepTest, DAKO) and fluorescence in situ hybridization (FISH). Thus, the cases were selected non-randomly.

In this feasibility study, we focused on the protein kinases ERK1/2 (extracellular signal-regulated kinase 1 and 2) and their dual phosphorylation on Thr202/Tyr204. First, we wished to confirm the specificity of the antibodies using extracts from breast cancer samples, since highly specific antibodies are an essential prerequisite for reverse phase protein microarrays. Furthermore, there may be differences in antibody specificity between cell lines and human tissues. Proteins were extracted from FFPE breast cancer samples and subjected to 1D gel electrophoresis and Western blot analysis. Fig. (2) shows that the antibodies used for detection of either ERK1/2 or phospho-ERK1/2, reacted only with proteins at about 42 and $44 \mathrm{kDa}$, corresponding to the presumed molecular weights of the p42 and p44 MAP kinases. No other band appeared in the Western blot, apparently demonstrating no cross-reactivities of the antibodies used. For HER2, we detected the known variants, p185 and p95, in HER2 positive cases. Beta-actin immunoreactivity was visualized as loading control, demonstrating that enough protein was loaded in the lane for the HER2 negative case.

Subsequently, we extracted proteins from 20 breast cancers and performed reverse phase protein microarray analysis in order to quantify ERK1/2, phospho-ERK1/2, and HER2 expression (Fig. 3). 


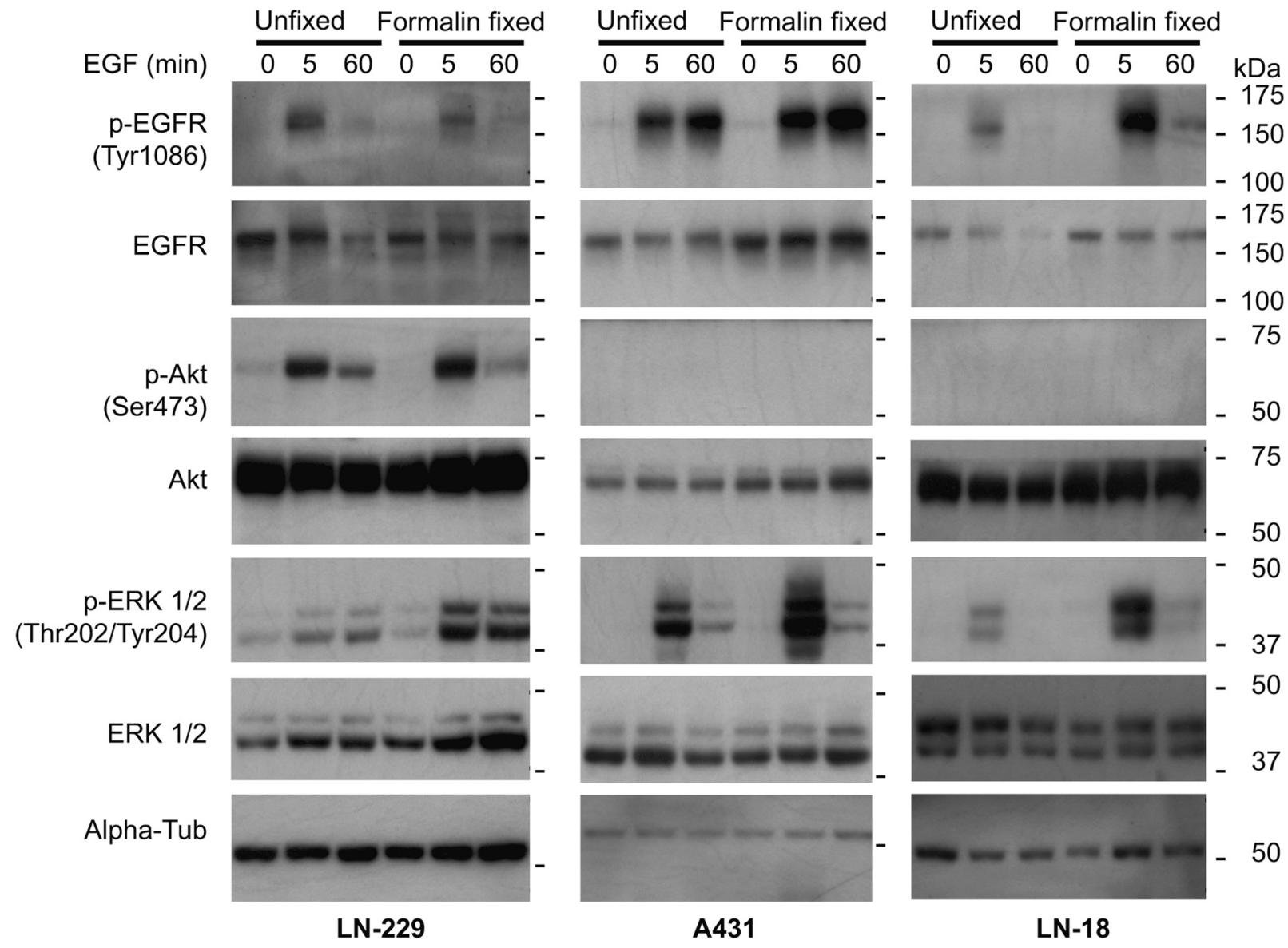

Fig. (1). Comparison of protein extraction efficiency between formalin-fixed and unfixed cells. Three epidermal growth factor receptor positive cell lines (LN-229, A431, LN-18) were treated with epidermal growth factor for 0, 5, and 60 minutes. Proteins were extracted and used for Western blot analysis. The proteins analyzed are indicated on the left. Molecular weights (in $\mathrm{kDa}$ ) are shown on the right. Phosphorylated proteins can be extracted after formalin fixation. The general time-course pattern is similar between unfixed and fixed cells.

A

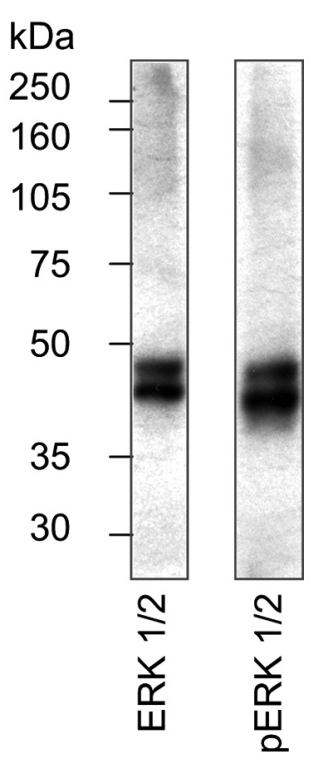

B

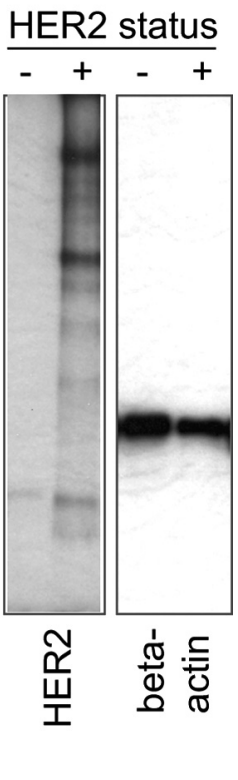

Fig. (2). Demonstration of antibody specificity using protein extracts from formalin-fixed and paraffin-embedded human tissues. Western blots for (A) ERK1/2, phospho-ERK1/2 (Thr202, Tyr204) and (B) HER2, beta-actin are shown. HER2 status: -, HER2 negative; +, HER2 positive.

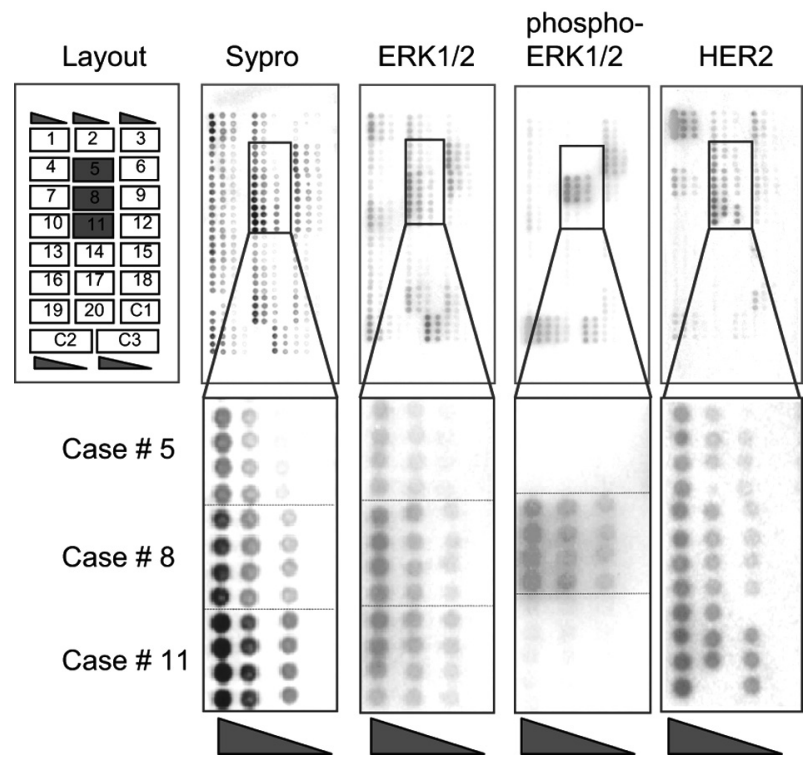

Fig. (3). Protein microarray analysis from extracts of 20 breast cancer samples. The cases \#5, \#8, and \#11 are shown in higher magnification as examples. Sypro, Sypro Ruby total protein stain. Samples labelled "C" are controls: C1, protein mixture composed of $1 \mu \mathrm{l}$ from each of the 20 patient protein lysates; $\mathrm{C} 2$, protein lysate from A431 cells; C3, protein lysate from LN-229 cells. 
Three cases (\#5, \#8, and \#11) are shown in detail as examples. The relative protein expressions from the three proteins normalized to total protein (Sypro Ruby intensity) are listed in Table 2. As shown in Table 3, expression of HER2 shows a statistically significant positive correlation with ERK1/2 $(p<0.03$, rho=0.495). However, no association $(p=0,95$, rho $=0.015)$ was observed between HER 2 and phospho-ERK1/2.

Table 2. Results from Reverse Phase Protein Microarrays. The Numbers Indicate Relative Protein Expression Levels Normalized to the Total Protein

\begin{tabular}{|c|c|c|c|}
\hline CASE \# & HER2 & ERK1/2 & PHOSPHO-ERK1/2 \\
\hline 1 & 0,73 & 0,41 & 0,13 \\
\hline 2 & 0,23 & 0,57 & 0,16 \\
\hline 3 & 1,18 & 0,66 & 1,52 \\
\hline 4 & 0,08 & 0,21 & 0,17 \\
\hline 5 & 0,48 & 0,50 & 0,04 \\
\hline 6 & 0,16 & 0,69 & 0,94 \\
\hline 7 & 0,39 & 0,09 & 0,14 \\
\hline 8 & 0,40 & 0,65 & 0,99 \\
\hline 9 & 0,23 & 0,63 & 0,27 \\
\hline 10 & 0,06 & 0,65 & 0,26 \\
\hline 11 & 0,45 & 0,47 & 0,05 \\
\hline 12 & 0,12 & 0,11 & 0,01 \\
\hline 13 & 0,08 & 0,22 & 0,09 \\
\hline 14 & 0,00 & 0,04 & 0,27 \\
\hline 15 & 0,00 & 0,15 & 0,36 \\
\hline 16 & 0,12 & 0,23 & 0,07 \\
\hline 17 & 0,00 & 0,26 & 0,14 \\
\hline 18 & 0,02 & 0,10 & 0,14 \\
\hline 19 & 0,14 & 0,41 & 0,15 \\
\hline 20 & 0,01 & 0,49 & 0,04 \\
\hline
\end{tabular}

Table 3. Results of Correlation Between HER2, ERK1/2, and Phospho-ERK1/2 Using Spearman Rank Correlation Test

\begin{tabular}{|c|c|c|}
\hline \multirow{2}{*}{} & \multicolumn{2}{|c|}{ HER2 } \\
\cline { 2 - 3 } & $\boldsymbol{P}$ & RHO \\
\hline \hline ERK1/2 & $<0.03$ & 0.495 \\
\hline PHOSPHO-ERK1/2 & 0.95 & 0.015 \\
\hline
\end{tabular}

\section{DISCUSSION}

A common mechanism for intracellular signal transmission consists of reversible phosphorylation and dephosphorylation by kinases and phosphatases, respectively. The development of novel proteomic technologies that can quantitatively monitor the phosphorylation states of signaling pro- teins in a multiplex fashion will be an important step towards unravelling the complex signaling networks in diseased tissues.

Reverse phase protein microarrays are the method of choice for the quantification of diagnostic and therapeutic protein targets because they use internal standard curves, have a low detection limit, are economical, and are highly multiplex [15-21]. This form of protein microarray has often been used for the quantification of phosphorylated proteins extracted from cell lines or frozen tissues. However, direct clinical applications for profiling signaling molecules are still at a very early stage.

As cancer results from deregulation of the cell's signaling network, clinical tissue research has a special interest in investigating the phosphorylation state of proteins. The vast majority of clinical tissue samples, however, are routinely fixed with formalin and embedded into paraffin (FFPE) to be conserved for histophathological examinations. This procedure causes alterations in cellular macromolecules, including protein cross-linking, and thus impedes extraction-based biochemical characterization. Recently, we developed a protocol allowing isolation of high amounts of full-length, nondegraded, and immunoreactive proteins from routinely formalin-fixed tissue specimen for Western blot and reverse phase protein microarray analyses [11]. Thus, the objective of this translational study was to clarify whether phosphorylated proteins can be extracted and quantified from formalinfixed tissues.

Extraction of phosphorylated proteins was compared between cultured cells of three different EGFR-positive cell lines that had been treated with EGF for various times and were either fixed with formalin or left unfixed. Lysates were analyzed by Western blot; three representative target proteins covering different kinds of phosphorylation in mammalian signal transduction pathways, phospho-EGFR, phospho-Akt and phospho-ERK1/2, could readily be detected. Although not identical, the general time-course pattern is similar between formalin-fixed and unfixed cells.

Subsequently, this novel method for analyzing phosphorylated proteins in formalin-fixed samples was applied in a first clinically relevant study investigating a central protein kinase in 20 breast cancers that had been selected based on HER2 status. HER2 is a receptor tyrosine kinase, the biological and clinical role of which has been extensively studied in breast cancer. There are two major signaling pathways downstream of the HER-family of receptors: the Raf-MEKERK pathway and the PI3K-Akt pathway [22, 23]. Here we focused on the activation (phosphorylation) of ERK1/2. In a previous report it was shown, that expression of the phosphorylated ERK $1 / 2$ proteins as determined by Western blot corresponded to the activity levels of the enzymes, as determined by a protein kinase assay [24]. We did not find a correlation between HER2 over-expression and activation of ERK1/2. These results are in line with immunohistochemical studies reported previously, using a total of 245 breast tumour samples $[24,25]$. In our study, we did not compare immunohistochemistry with protein microarray analysis because there is no standardized evaluation score available for ERK1/2 and phospho-ERK1/2. 
In future studies, analysis of additional HER receptors (e.g., EGF receptor) and their phosphorylated forms may be included in order to establish the significance of HER receptor activation and phosphorylation of downstream signaling molecules in breast cancer. Generally, our approach could be the basis for the design of novel combination therapies for a targeted treatment of signaling pathways in a variety of cancers (Fig. 4). Moreover, analyzing signaling pathways in FFPE tissues may be useful to predict response of novel antiHER antibody-based therapies in breast and colorectal cancers.

\section{CONCLUSIONS}

Taken together, this proof-of-principle study supports the hypothesis that every tumour exhibits a unique combination of molecular alterations which requires detection of a sufficient number of phosphorylated signal transduction proteins in order to develop patient-tailored therapies targeting deregulated signaling pathways. The successful utility of reverse phase protein microarrays for analysis of phosphorylated proteins in formalin-fixed tissue samples has been demonstrated.

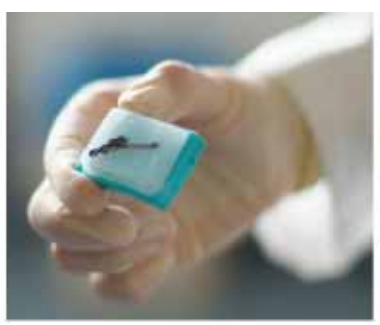

FFPE tissue

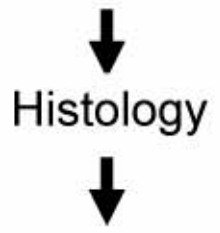

Protein extraction

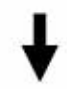

Protein lysate

microarrays

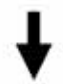

Quantitation of deregulated signalling pathways

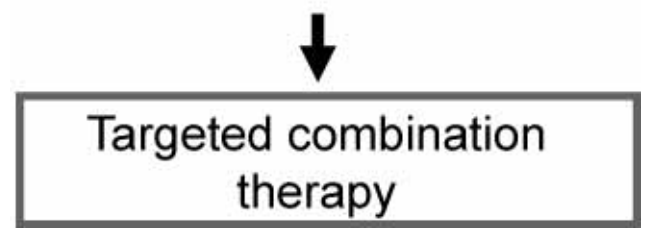

Fig. (4). Vision for a possible clinical application of our approach. Monitoring deregulated signaling pathways in formalin-fixed and paraffin-embedded (FFPE) tissue samples may be used to identify possible target structures for individualized cancer treatments.

\section{ACKNOWLEDGEMENT}

The authors want to thank Tibor Schuster, Dr. Jürgen Schlegel, and the group members of IMPACTS (Integration of Molecular Pathology and Cell and Tissues Structure) for discussions. The study was supported by a grant from the Deutsche Forschungsgemeinschaft (DFG) to KFB and $\mathrm{HH}$ (Grant No. BE 1501/3-2).

\section{CONFLICT OF INTEREST}

$\mathrm{KFB}$ is a named inventor of a patent related to protein extraction from formalin fixed tissues. He had full access to all of the data in this study and takes complete responsibility for the integrity of the data and the accuracy of the data analysis. Qiagen $\mathrm{GmbH}$., the owner of the patent, did not assert control over the authors' right to publish.

\section{REFERENCES}

[1] Cowherd SM, Espina VA, Petricoin EF 3rd, Liotta LA. Proteomic analysis of human breast cancer tissue with laser-capture microdissection and reverse-phase protein microarrays. Clin Breast Cancer 2004; 5: 385-92.

[2] Dhanasekaran DN, Johnson GL. MAPKs: function, regulation, role in cancer and therapeutic targeting. Oncogene 2007; 26: 3097-99.

[3] Raman M, Chen W, Cobb MH. Differential regulation and properties of MAPKs. Oncogene 2007; 26: 3100-12.

[4] Gulmann C, Sheehan KM, Kay EW, Liotta LA, Petricoin EF 3rd. Array-based proteomics: mapping of protein circuitries for diagnostics, prognostics, and therapy guidance in cancer. J Pathol 2006; 208: 595-606.

[5] Liotta LA, Kohn EC, Petricoin EF. Clinical proteomics: personalized molecular medicine. JAMA 2001; 286: 2211-14.

[6] Wulfkuhle JD, Edmiston KH, Liotta LA, Petricoin EF 3rd. Technology insight: pharmacoproteomics for cancer-promises of patient-tailored medicine using protein microarrays. Nat Clin Pract Oncol 2006; 3: 256-68.

[7] Dancey J, Sausville EA. Issues and progress with protein kinase inhibitors for cancer treatment. Nat Rev Drug Discov 2003; 2: 296313.

[8] Dancey JE, Chen HX. Strategies for optimizing combinations of molecularly targeted anticancer agents. Nat Rev Drug Discov 2006; 5: 649-59.

[9] Dietel M, Sers C. Personalized medicine and development of targeted therapies: The upcoming challenge for diagnostic molecular pathology. A review. Virchows Arch 2006; 448: 744-55.

[10] Becker KF, Metzger V, Hipp S, Höfler H. Clinical proteomics: new trends for protein microarrays. Curr Med Chem 2006; 13: 1831-37. Becker KF, Schott C, Hipp S, et al. Quantitative protein analysis from formalin-fixed tissues: implications for translational clinical research and nanoscale molecular diagnosis. J Pathol 2007; 211: 370-78.

[12] Haubner R, Weber WA, Beer AJ, et al. Noninvasive visualization of the activated alphavbeta3 integrin in cancer patients by positron emission tomography and $[18$ F]Galacto-RGD. PLoS Med 2005; 2 : e70

[13] Schlegel J, Piontek G, Budde B, Neff F, Kraus A. The Akt/protein kinase B-dependent anti-apoptotic pathway and the mitogenactivated protein kinase cascade are alternatively activated in human glioblastoma multiforme. Cancer Lett 2000; 158: 103-8.

Becker KF, Schott C, Becker I, Höfler H. Guided protein extraction from formalin-fixed tissues for quantitative multiplex analysis avoids detrimental effects of histological stains. Proteomics Clin Appl 2008, in press, DOI 10.1002/prca. 200780106.

Espina V, Mehta AI, Winters ME, et al. Protein microarrays: molecular profiling technologies for clinical specimens. Proteomics 2003; 3: 2091-2100.

Paweletz CP, Charboneau L, Bichsel VE, et al. Reverse phase protein microarrays which capture disease progression show activation of pro-survival pathways at the cancer invasion front. Oncogene 2001; 20: 1981-89.

[17] Liotta LA, Espina V, Mehta AI, et al. Protein microarrays: meeting analytical challenges for clinical applications. Cancer Cell 2003; 3: $317-25$. 
[18] Chan SM, Ermann J, Su L, Fathman CG, Utz PJ. Protein microarrays for multiplex analysis of signal transduction pathways. Nat Med 2004; 10: 1390-96.

[19] Grubb RL, Calvert VS, Wulkuhle JD, et al. Signal pathway profiling of prostate cancer using reverse phase protein arrays. Proteomics 2003; 3: 2142-46.

[20] Hipp S, Walch A, Schuster T, Höfler H, Becker KF. Precise measurement of the E-cadherin repressor Snail in formalin-fixed endometrial carcinoma using protein lysate microarrays. Clin Exp Metastasis 2008; Feb 29 [Epub ahead of print].

[21] Tibes R, Qiu Y, Lu Y, et al. Reverse phase protein array: validation of a novel proteomic technology and utility for analysis of primary leukemia specimens and hematopoietic stem cells. Mol Cancer Ther 2006; 5: 2512-21.
[22] Hynes NE, Lane HA. ERBB receptors and cancer: the complexity of targeted inhibitors. Nat Rev Cancer 2005; 5: 341-54.

[23] McKay MM, Morrison DK. Integrating signals from RTKs to ERK/MAPK. Oncogene 2007; 26: 3113-21.

[24] Mueller H, Flury N, Eppenberger-Castori S, Kueng W, David F, Eppenberger U. Potential prognostic value of mitogen-activated protein kinase activity for disease-free survival of primary breast cancer patients. Int J Cancer 2000; 89: 384-88.

[25] Svensson S, Jirström K, Rydén L, et al. ERK phosphorylation is linked to VEGFR2 expression and Ets-2 phosphorylation in breast cancer and is associated with tamoxifen treatment resistance and small tumours with good prognosis. Oncogene 2005; 24: 4370-79.

(C) Becker et al.; Licensee Bentham Open.

This is an open access article distributed under the terms of the Creative Commons Attribution License (http://creativecommons.org/licenses/by/2.5/), which permits unrestrictive use, distribution, and reproduction in any medium, provided the original work is properly cited. 